\title{
Investigating the Gaussian Convergence of the Distribution of the Aggregate Interference Power in Large Wireless Networks
}

\author{
Muhammad Aljuaid and Halim Yanikomeroglu, Member, IEEE
}

\begin{abstract}
The distribution of the aggregate interference power in large wireless networks has gained increasing attention with the emergence of different types of wireless networks such as ad hoc networks, sensor networks, and cognitive radio networks. The interference in such networks is often characterized using the Poisson point process (PPP). As the number of interfering nodes increases, there might be a tendency to approximate the distribution of the aggregate interference power by a Gaussian random variable, given that the individual interference signals are independent. However, some observations in the literature suggest that this Gaussian approximation is not valid, except under some specific scenarios. In this paper, we cast these observations in a single mathematical framework and express the conditions for which the Gaussian approximation will be valid for the aggregate interference power generated by a Poisson field of interferers. Furthermore, we discuss the effect of different system and channel parameters on the convergence of the distribution of the aggregate interference to a Gaussian distribution.
\end{abstract}

Index Terms-Berry-Esseen bound, cochannel interference, cumulants, fading, Poisson point process (PPP).

\section{INTRODUCTION}

$\mathbf{T}$ HE INTEREST in characterizing the distribution of the aggregate interference power in large wireless networks has increased with the emergence of different types of wireless networks. Examples of these networks include wireless sensor networks, ad hoc networks [2], [3], and cognitive radio networks [4], [5].

It is common to characterize the interference in large wireless networks using the Poisson point process (PPP). Therefore, the aggregate interference can be considered as the sum of a large number of independent interference signals. Thus, there might be a tendency to approximate the aggregate interference power by a Gaussian random variable. However, this approximation is

Manuscript received December 21, 2009; revised May 21, 2010; accepted July 20, 2010. Date of publication August 16, 2010; date of current version November 12, 2010. This work was supported in part by Saudi Aramco, Dhahran, Saudi Arabia. This paper was presented in part at the 25th Biennial Symposium on Communications, Queen's University, Kingston, ON, Canada, May 12-14, 2010 [1]. The review of this paper was coordinated by Prof. G. Bauch.

M. Aljuaid was with Carleton University, Ottawa, ON K1S 5B6, Canada. He is now with Saudi Aramco, Dhahran 31311, Saudi Arabia (e-mail: mjuaid@gmail.com).

H. Yanikomeroglu is with the Department of Systems and Computer Engineering, Carleton University, Ottawa, ON K1S 5B6, Canada (e-mail: halim@sce.carleton.ca).

Color versions of one or more of the figures in this paper are available online at http://ieeexplore.iee.org.

Digital Object Identifier 10.1109/TVT.2010.2067452 not valid, except under certain conditions. The authors in [6] consider the interference in a code-division multiple-access (CDMA) network and indicate that the distribution of the aggregate interference power from users in other cells is likely to be Gaussian if there are a large number of interfering users in the vicinity of the victim cell. The authors in [7] also consider the aggregate interference in a CDMA network and show that the distribution of the aggregate interference power converges to a Gaussian distribution as the traffic measure (which can be related to the average number of interferers in a cell) goes to infinity. The authors in [5] indicate that the central limit theorem $(\mathrm{CLT})^{1}$ does not apply in the case where some of the interferers are dominant, although the number of interferers may be large. It is indicated in [2] that the Gaussian distribution is a bad approximation for the distribution of the aggregate interference when the node density is low. Based on simulation results, Hasan and Andrews [9] show that the Gaussian approximation could be acceptable when there is a wide-enough exclusion region (with no interferers) around the victim receiver. It is also indicated in [10] that, when the exclusion region is relatively small, the distribution of the interference power has a heavy tail; therefore, the Gaussian distribution does not model the interference very well.

Observing that the aggregate interference can be modeled as shot noise, discussions in [11] on the convergence of shot noise to a Gaussian random variable become relevant to our study. The authors in [11] proved that, under certain conditions, the shot noise converges in distribution to Gaussian when the intensity (density) of the underlying point process of the shot noise goes to infinity. However, no discussion has been given to the effect of the exclusion region on this convergence. In this paper, we apply some of the results obtained in [11] to the case of the aggregate interference power in large wireless networks. We incorporate in the formulations the effect of the exclusion region and identify the rate of the Gaussian convergence with respect to the size of the exclusion region. Moreover, we discuss the effect of fading distributions, including small- and largescale fading, on this convergence.

This paper is organized as follows: Section II describes the system model. Then, Section III establishes the mathematical framework to quantify how far away the distribution of the aggregate interference power is from a Gaussian distribution. Moreover, the same section discusses some system and channel parameters affecting the convergence to a Gaussian distribution.

\footnotetext{
${ }^{1}$ The history of the CLT is summarized in [8].
} 


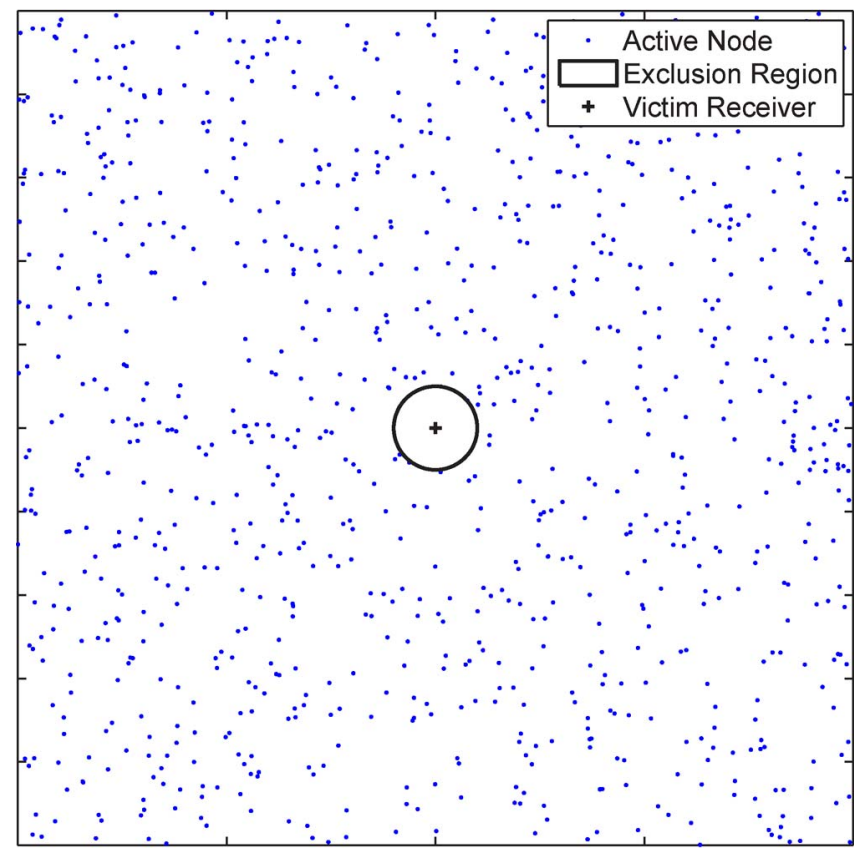

Fig. 1. Realization of the spatial distribution of (blue dots) active nodes. (Plus sign) Victim receiver. (Circle) Boundary of an exclusion region of radius $r_{o}$.

The effect of the fading distributions is addressed in Section IV. This paper is concluded by some remarks in Section V.

\section{SYSTEM MODEL}

In this paper, we model a large wireless network by an infinite random field of interferers (active nodes). These active nodes are distributed over $\mathbb{R}^{2}$, which is a 2-D Euclidean space, ${ }^{2}$ according to a $\mathrm{PPP}^{3}$ with an intensity (density) of $\lambda>0$. A victim receiver is assumed to be located within the field. There might be an exclusion region of radius $r_{o}<\infty$ around this victim receiver in which there are no active nodes, as illustrated in Fig. 1.

The victim receiver receives an interference power $I_{i}$ from the transmission of an active node $i$. Under the assumption of incoherent addition of the interfering signals, the aggregate interference power received by the victim receiver can be expressed as

$$
I_{A}=\sum_{i \in \Lambda} I_{i}=\sum_{i \in \Lambda} X_{i} g\left(r_{i}\right)
$$

where $\Lambda$ is the set of active nodes, and $X_{i}$ is a random variable resulting from the multiplication of some deterministic parameters and random variables. Without loss of generality, we assume the deterministic parameters in $X_{i}$ to be equal to 1 . Therefore, we consider $X_{i}$ as a random variable modeling small-scale, large-scale, or composite fading. The $X_{i}$ 's values are assumed to be independent and identically distributed (i.i.d.), which is a common assumption in similar works such as [5], [9], and [12]. The function $g\left(r_{i}\right)$ models the

\footnotetext{
${ }^{2}$ Appendix $\mathrm{C}$ extends some of the results obtained in this paper for a $d$-dimensional space.

${ }^{3}$ For the justification of using PPP for node distribution in wireless networks, see [5].
}

distance-dependent attenuation, where $r_{i}$ is the Euclidean distance between node $i$ and the victim receiver. In the rest of this paper, we simply call $g\left(r_{i}\right)$ the path loss.

Some path-loss models used in the literature suffer from a singularity at $r_{i}=0$, which may affect the performance measures of the wireless network [13]. Therefore, we consider in our analysis the following nonsingular model [14]:

$$
g\left(r_{i}\right)= \begin{cases}\alpha r_{i}^{-n}, & r_{i} \geq r_{c} \\ \alpha r_{c}^{-n}, & r_{i}<r_{c}\end{cases}
$$

where $r_{c}>0$ is a critical distance below which $g\left(r_{i}\right)$ becomes a constant. The constant parameter $\alpha$ can be assumed to be 1 without loss of generality. The path-loss exponent is denoted by $n$ and assumed to be greater than 2 .

\section{BERRY-ESSEEN BOUND FOR THE DISTRIBUTION OF $I_{A}$}

Our investigation of the Gaussianity of the distribution of $I_{A}$ is based on the Berry-Esseen bound. This bound is explained in its basic form in Appendix A. Formulations for the Berry-Esseen bound when the underlying process is a stationary PPP are obtained in [11]. These formulations are extended in [15] by removing the requirement that a PPP is stationary. We apply the results in [11] and [15] to investigate the Gaussianity of the distribution of $I_{A}$.

Let $\tilde{\mu}_{A}$ and $\sigma_{A}$ be the mean and standard deviation of $I_{A}$, respectively. Let $F_{Z}(y)$ denote the cumulative distribution function (cdf) of $Z$ (where $Z=\left(I_{A}-\tilde{\mu}_{A}\right) / \sigma_{A}$ ), and let $F_{N}$ denote the cdf of the standard normal distribution, i.e., $\mathcal{N}(0,1)$. Then, for all $y$

$$
\left|F_{Z}(y)-F_{N}(y)\right| \leq 2.21 \frac{\kappa_{3}\left(I_{A}\right)}{\left[\kappa_{2}\left(I_{A}\right)\right]^{\frac{3}{2}}}
$$

where $\kappa_{2}\left(I_{A}\right)$ and $\kappa_{3}\left(I_{A}\right)$ are the second and third cumulants of $I_{A}$, respectively. The formulation of the Berry-Esseen bound in [11] is not explicitly presented in terms of cumulants. However, as shown in Appendix B, we equivalently expressed the Berry-Esseen bound using the second and third cumulants.

Results obtained in [10], [16], and [17] can be used to find $\kappa_{2}\left(I_{A}\right)$ and $\kappa_{3}\left(I_{A}\right){ }^{4}$ Based on [16], the $m$ th cumulant of $I_{A}$ can be written as

$$
\kappa_{m}\left(I_{A}\right)=N_{\text {eff }}(m) g\left(r_{o}\right)^{m} \tilde{\mu}_{m}(X)
$$

where

$$
\begin{aligned}
N_{\text {eff }}(m) & =\lambda \pi\left[r_{\text {eff }}^{2}(m)-r_{o}^{2}\right] \\
r_{\text {eff }}(m) & =\max \left(r_{c}, r_{o}\right) \sqrt{\frac{m n}{m n-2}} .
\end{aligned}
$$

Here, $N_{\text {eff }}(m)$ virtually represents the average number of active nodes as seen by the victim receiver in an annular region of

\footnotetext{
${ }^{4}$ It is worth highlighting that cumulants are derived by differentiating the logarithm of the characteristic function (or the moment-generating function) of $I_{A}$. There are different approaches in the literature to derive this characteristic function for a PPP. One approach is to use the conditional expectation, as explained in [10] and [12]. Another approach is to use Campbell's theorem for a PPP, as demonstrated in [4], [6], and [16].
} 
inner radius $r_{o}$ and outer radius $r_{\text {eff }}$. The parameter $\tilde{\mu}_{m}(X)$ denotes the $m$ th raw moment of $X_{i}$, i.e., $\mathrm{E}\left[X_{i}^{m}\right]$. For convenience and since $X_{i}$ 's are i.i.d., we omit the subscript $i$. Using (4) and (5) in (3), the Berry-Esseen bound for the distribution of $I_{A}$ can be expressed as

$$
\left|F_{Z}(y)-F_{N}(y)\right| \leq 2.21 \frac{N_{\text {eff }}(3) \tilde{\mu}_{3}(X)}{\left[N_{\text {eff }}(2) \tilde{\mu}_{2}(X)\right]^{\frac{3}{2}}} .
$$

There are three possible topologies with respect to the exclusion region: 1) an exclusion region with $r_{o} \geq r_{c}$;2) no exclusion region $\left(r_{o}=0\right)$; and 3$)$ an exclusion region with $0<r_{o}<r_{c}$. We do not discuss the third topology here due to space limitations and since the results for the third topology are bounded by the results of the first two topologies.

1) Exclusion Region $\left(r_{o} \geq r_{c}\right)$ : For this topology, it can be shown from (5) and (6) that the Berry-Esseen bound yields

$$
\left|F_{Z}(y)-F_{N}(y)\right| \leq 2.21 \frac{2(n-1)^{\frac{3}{2}}}{3 n-2} \frac{1}{\sqrt{\lambda \pi r_{o}^{2}}} \frac{\tilde{\mu}_{3}(X)}{\left[\tilde{\mu}_{2}(X)\right]^{\frac{3}{2}}} .
$$

\section{Remarks:}

1) It is observed from (7) that the bound is mainly controlled by the path-loss exponent, the active node density, the radius of the exclusion region, and the fading distribution.

2) The active node density is an important parameter in the convergence of the distribution of $I_{A}$ to a Gaussian distribution. As $\lambda$ increases, the bound becomes smaller, and the distribution of $I_{A}$ becomes closer to the Gaussian distribution; Figs. 2 and 3 show simulation results supporting this remark. Fig. 2 considers the histograms of the normalized $I_{A}$ for different values of $\lambda$. These histograms reflect the shape of the probability density function (pdf) of $I_{A}$, as compared with a Gaussian distribution. In Fig. 3, the comparison is performed among the cdf's. The rest of the figures shown in this paper focus on cdf's, which are more relevant to the Berry-Esseen bound. It is observed from (7) that the rate of Gaussian convergence with respect to the increase in $\lambda$ is $\sqrt{\lambda}$, which agrees with the findings in [11].

3) Similarly, as the exclusion region increases, the bound in (7) becomes smaller. Hence, the distribution of $I_{A}$ converges to a Gaussian distribution. Simulation results shown in Fig. 4 demonstrate this convergence. However, the convergence caused by increasing $r_{o}$ is faster than the convergence caused by increasing $\lambda$. The rate of convergence with respect to the size of the exclusion region is $\sqrt{\pi r_{o}^{2}}$.

4) An explanation for this convergence with respect to the increase in $\lambda$ and $r_{o}$ is as follows: What really matters for the convergence of the distribution of $I_{A}$ to Gaussianity is the number of dominant interferers around the victim receiver and not the total number of interferers in the field. The number of dominant interferers is mainly controlled by the active node density and an effective area around the victim receiver. As $\lambda$ increases, the number of dominant interferers increases. Similarly, as $r_{o}$ increases, the effective area increases as well; hence, the number of

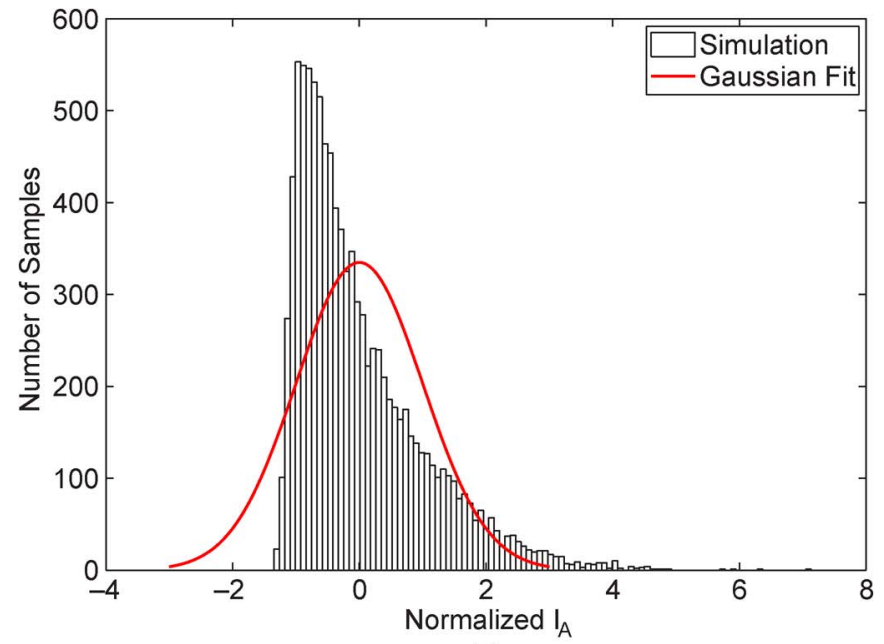

(a)

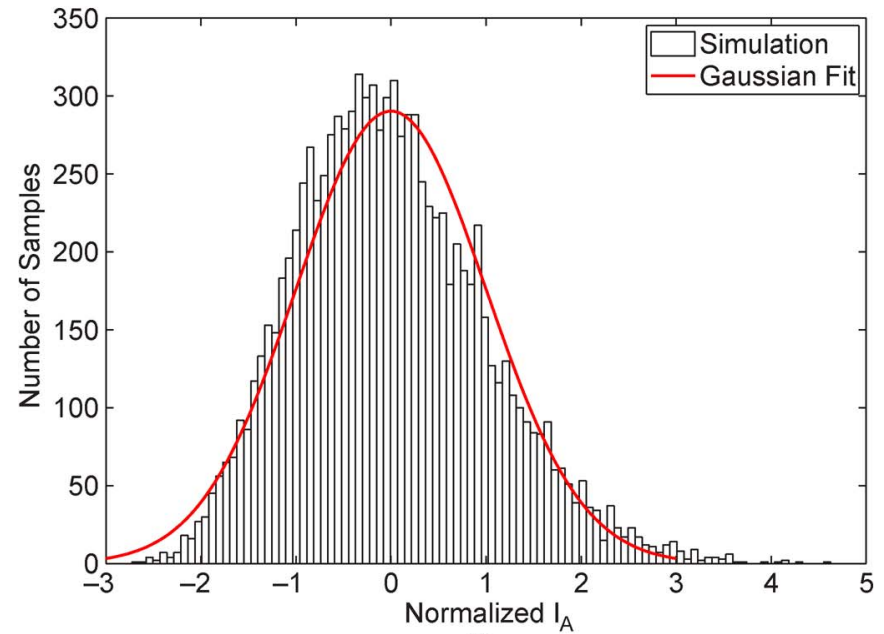

(b)

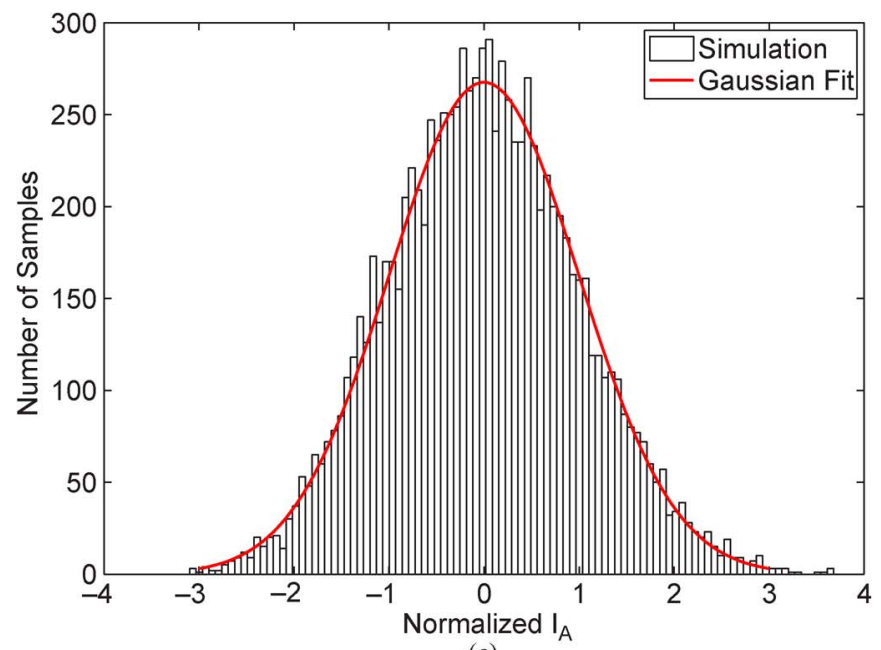

(c)

Fig. 2. Monte Carlo simulation-based histograms for the normalized $I_{A}$, i.e., $\left(I_{A}-\tilde{\mu}_{A}\right) / \sigma_{A}$, for different values of $\lambda\left(r_{o}=10 \mathrm{~m}, r_{c}=1 \mathrm{~m}, n=3\right.$, no multipath fading, and no shadow fading). (a) $\lambda=0.001$ nodes $/ \mathrm{m}^{2}$. (b) $\lambda=$ 0.01 nodes $/ \mathrm{m}^{2}$. (c) $\lambda=0.1$ nodes $/ \mathrm{m}^{2}$.

the dominant interferers increases. By virtue of the CLT, as the number of the dominant interferers increases, the distribution of $I_{A}$ converges to a Gaussian distribution.

5) The lower $n$ is, the better the convergence becomes. However, this effect on convergence is minor since the 


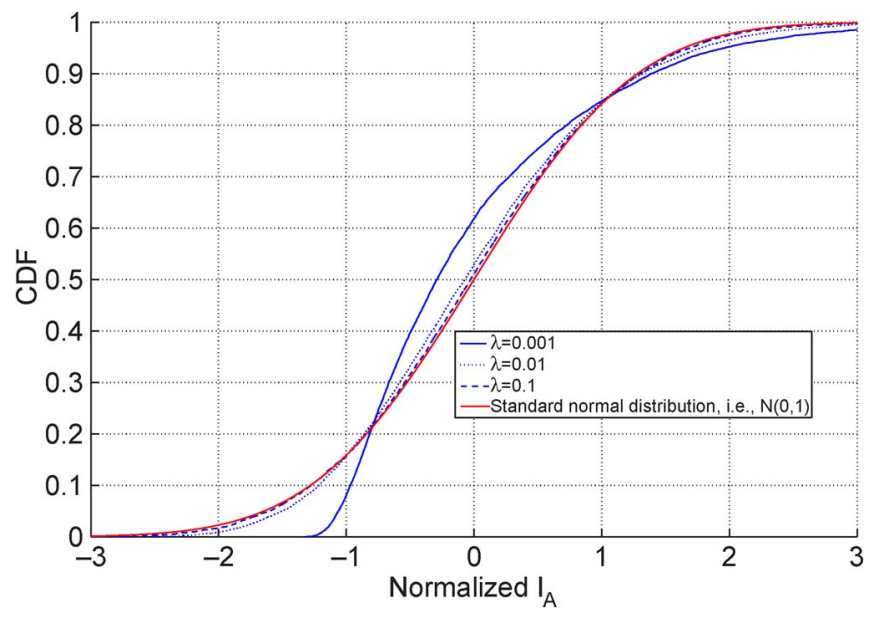

Fig. 3. Monte Carlo simulation-based cdf for the normalized $I_{A}$, i.e., $\left(I_{A}-\right.$ $\left.\tilde{\mu}_{A}\right) / \sigma_{A}$, for different values of $\lambda\left(r_{o}=10 \mathrm{~m}, r_{c}=1 \mathrm{~m}\right.$, and $\left.n=3\right)$.

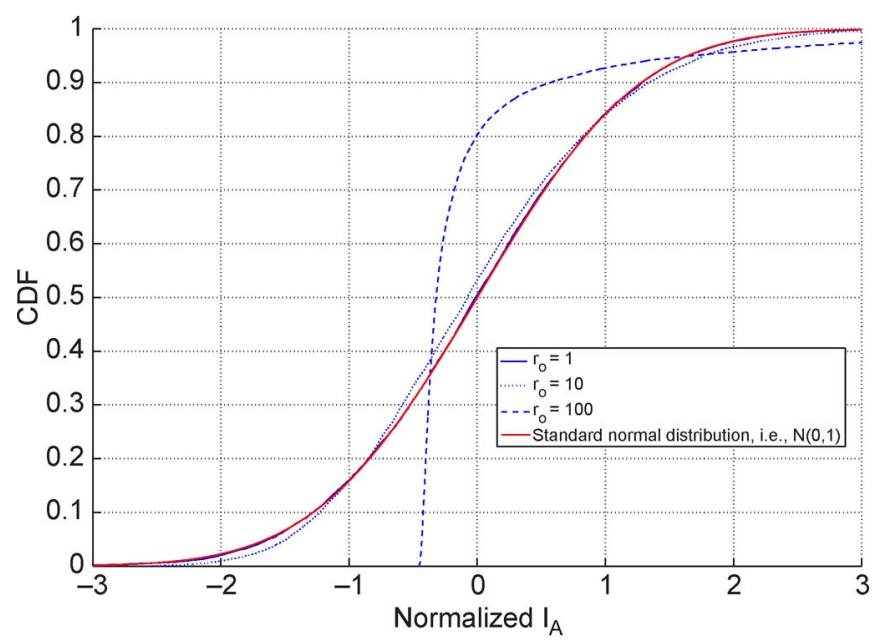

Fig. 4. Monte Carlo simulation-based cdf for the normalized $I_{A}$, i.e., $\left(I_{A}-\right.$ $\left.\tilde{\mu}_{A}\right) / \sigma_{A}$, for different values of $r_{o}\left(\lambda=0.01\right.$ nodes $/ \mathrm{m}^{2}, r_{c}=1 \mathrm{~m}$, and $\left.n=3\right)$.

range of $n$ is practically limited. Assuming $n \in(2,6]$

$$
0.5<\frac{2(n-1)^{\frac{3}{2}}}{3 n-2} \leq 1.4 \text {. }
$$

The effect of the fading distribution on the Gaussianity of $I_{A}$ is discussed in Section IV.

2) No Exclusion Region $\left(r_{o}=0\right)$ : If there is no exclusion region around the victim receiver, i.e., $r_{o}=0$, then it can be shown from (5) and (6) that the Berry-Esseen bound becomes

$$
\left|F_{Z}(y)-F_{N}(y)\right| \leq 2.21 \frac{3(n-1)^{\frac{3}{2}}}{\sqrt{n}(3 n-2)} \frac{1}{\sqrt{\lambda \pi r_{c}^{2}}} \frac{\tilde{\mu}_{3}(X)}{\left[\tilde{\mu}_{2}(X)\right]^{\frac{3}{2}}} .
$$

\section{Remarks:}

1) From (9), it might be concluded that a high $r_{c}$ value improves the Gaussian approximation. However, $r_{c}$ is used in the path-loss model to avoid the singularity at $r_{i}=$ 0 . The model effectively fixes the distance-dependent attenuation over a small disk of radius $r_{c}$ around the victim receiver. Therefore, the value of $r_{c}$ should be kept relatively small.
2) To justify the Gaussian approximation for this topology, $\sqrt{\lambda \pi r_{c}^{2}}$ should be large. However, since $r_{c}$ is relatively small, then $\lambda$ should be very high. To demonstrate this, let us assume that there are two networks, i.e., $\operatorname{Net}_{\left(r_{o}=100\right)}$ with an exclusion region of $r_{o}=100 \mathrm{~m}$ and $\mathrm{Net}_{\left(r_{o}=0\right)}$ without an exclusion region. Except for $r_{o}$, these two networks are assumed to have the same system and channel parameters, including $r_{c}=1 \mathrm{~m}$ and $n=3$. Assuming that these two networks have the same active node densities, the Berry-Esseen bound for $\operatorname{Net}_{\left(r_{o}=100\right)}$ can be shown to be smaller than that for $\operatorname{Net}_{\left(r_{o}=0\right)}$ by about $20 \mathrm{~dB}$. Therefore, the distribution of $I_{A}$ in $\operatorname{Net}_{\left(r_{o}=100\right)}$ is much closer to Gaussianity in comparison with the distribution of $I_{A}$ in $\operatorname{Net}_{\left(r_{o}=0\right)}$. For $\operatorname{Net}_{\left(r_{o}=0\right)}$ to have the same Berry-Esseen bound value as $\operatorname{Net}_{\left(r_{o}=100\right)}$, the active node density in $\operatorname{Net}_{\left(r_{o}=0\right)}$ has to be about $40 \mathrm{~dB}$ higher, which might be too high, unless the active node density of $\operatorname{Net}_{\left(r_{o}=100\right)}$ is too low.

3) Therefore, the Gaussian approximation could be possible for wireless networks with a sufficiently wide exclusion region. However, the validity of the approximation is questionable when there is no exclusion region or when the exclusion region is small, ${ }^{5}$ unless the active node density is very high, which might be practically infeasible.

4) From (7) and (9), it might be deduced that the Gaussian approximation is valid if

$$
\sqrt{\lambda \pi r_{o}^{2}} \gg 2.21 \frac{2(n-1)^{\frac{3}{2}}}{3 n-2} \frac{\tilde{\mu}_{3}(X)}{\left[\tilde{\mu}_{2}(X)\right]^{\frac{3}{2}}}, \quad \text { for } r_{o} \geq r_{c}
$$

or

$$
\sqrt{\lambda \pi r_{c}^{2}} \gg 2.21 \frac{3(n-1)^{\frac{3}{2}}}{\sqrt{n}(3 n-2)} \frac{\tilde{\mu}_{3}(X)}{\left[\tilde{\mu}_{2}(X)\right]^{\frac{3}{2}}}, \quad \text { for } r_{o}=0
$$

\section{EFFECT OF FADING DistRIBUTIONS ON THE GAUSSiAn ApProximation of $I_{A}$}

In this section, we investigate the effect of the distribution of $X_{i}$, i.e., the fading distribution, on the Gaussian convergence of the distribution of $I_{A}$. We provide expressions for $\tilde{\mu}_{3}(X) /\left[\tilde{\mu}_{2}(X)\right]^{3 / 2}$ considering different fading cases. These expressions can be used with (6), (7), or (9) to get the related Berry-Esseen bounds.

\section{A. Case I: Without Multipath Fading and Without Shadow Fading}

For the case without fading (neither multipath nor shadow fading), $X_{i}$ becomes deterministic. Therefore

$$
\frac{\tilde{\mu}_{3}(X)}{\left[\tilde{\mu}_{2}(X)\right]^{\frac{3}{2}}}=1
$$

\footnotetext{
${ }^{5}$ The authors in [10] show that a lognormal distribution can be used to approximate the distribution of the aggregate interference power when the exclusion region is small.
} 
which leads to

$$
\left|F_{Z}(y)-F_{N}(y)\right|<2.21 \frac{2(n-1)^{\frac{3}{2}}}{3 n-2} \frac{1}{\sqrt{\lambda \pi r_{o}^{2}}} .
$$

Equation (13) may be considered as the baseline to judge the effect of fading on the Gaussian convergence.

\section{B. Case II: With Multipath Fading But Without Shadow Fading}

The effect of multipath fading on the received individual interference power $I_{i}$ can be modeled by a Gamma random variable (which is a result of the assumption that the envelope of the interference signal can be modeled by the versatile Nakagami distribution). In this case, the pdf of $X_{i}$ can be represented as [18]

$$
f_{X}(x)=\left(\frac{\nu}{\Omega}\right)^{\nu} \frac{x^{\nu-1}}{\Gamma(\nu)} e^{-\frac{\nu}{\Omega} x}, \quad x>0, \quad \nu \geq \frac{1}{2}
$$

where $\Omega$ is the average received power, $\nu$ is the shape parameter, and $\Gamma(\cdot)$ is the Gamma function. The $m$ th moment of $X_{i}$ can be expressed as

$$
\tilde{\mu}_{m}(X)=\left(\frac{\Omega}{\nu}\right)^{m} \frac{\Gamma(\nu+m)}{\Gamma(\nu)} .
$$

Thus

$$
\frac{\tilde{\mu}_{3}(X)}{\left[\tilde{\mu}_{2}(X)\right]^{\frac{3}{2}}}=\frac{\Gamma(\nu+3)}{[\Gamma(\nu+2)]^{\frac{3}{2}}} \Gamma(\nu)^{\frac{1}{2}}=\frac{\nu+2}{\sqrt{\nu(\nu+1)}} .
$$

We may conclude that multipath fading shifts the distribution of $I_{A}$ away from Gaussianity. However, the shift is limited since, for $(1 / 2) \leq \nu<\infty$

$$
1<\frac{\nu+2}{\sqrt{\nu(\nu+1)}} \leq 2.89
$$

\section{Case III: With Shadow Fading But Without Multipath Fading}

Shadow fading is commonly modeled by a lognormal random variable with logarithmic mean $0 \mathrm{~dB}$ and standard deviation $\sigma_{s} \mathrm{~dB}$. Therefore, the effect of shadow fading on the aggregate interference power can be reflected by assuming that $X_{i}$ is a lognormal random variable, and hence, it has the following expression for its $m$ th moment:

$$
\tilde{\mu}_{m}(X)=e^{\frac{1}{2}\left(m \frac{\ln 10}{10} \sigma_{s}\right)^{2}}
$$

which leads to

$$
\frac{\tilde{\mu}_{3}(X)}{\left[\tilde{\mu}_{2}(X)\right]^{\frac{3}{2}}}=e^{\frac{3}{2}\left(\frac{\ln 10}{10} \sigma_{s}\right)^{2}} .
$$

As shown in Fig. 5, for typical values of $\sigma_{s}$, e.g., $\sigma_{s} \in$ $[4,10] \mathrm{dB}$, the effect of shadow fading on $\tilde{\mu}_{3}(X) /\left[\tilde{\mu}_{2}(X)\right]^{3 / 2}$, and consequently on the Berry-Esseen bound, could be dominant, compared with the effect of multipath fading.

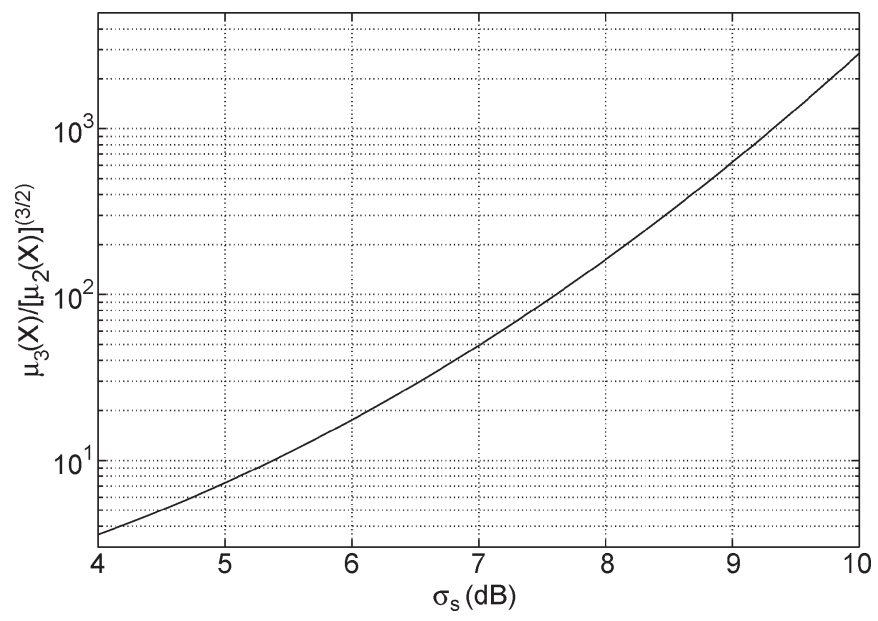

Fig. 5. Values of $\tilde{\mu}_{3}(X) /\left[\tilde{\mu}_{2}(X)\right]^{3 / 2}$ for different values of $\sigma_{s}$.

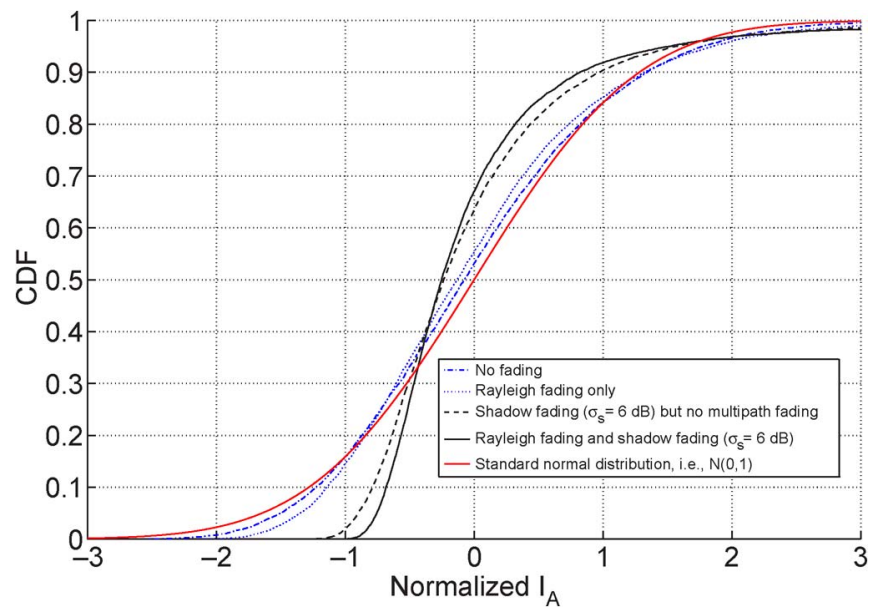

Fig. 6. Monte Carlo simulation-based cdf for the normalized $I_{A}$, i.e., $\left(I_{A}-\right.$ $\left.\tilde{\mu}_{A}\right) / \sigma_{A}$, for different fading scenarios $\left(r_{o}=10 \mathrm{~m}, r_{C}=1 \mathrm{~m}, n=3\right.$, and $\lambda=0.01$ nodes $/ \mathrm{m}^{2}$ ).

\section{Case IV: With Composite Fading}

If the interference signals are subject to both multipath and shadow fading, $X_{i}$ can be modeled as the product of two independent random variables, such as a Gamma random variable (for multipath fading) and a lognormal random variable (for shadow fading). Thus, using (16) and (19)

$$
\frac{\tilde{\mu}_{3}(X)}{\left[\tilde{\mu}_{2}(X)\right]^{\frac{3}{2}}}=\frac{\nu+2}{\sqrt{\nu(\nu+1)}} e^{\frac{3}{2}\left(\frac{\ln 10}{10} \sigma_{s}\right)^{2}} .
$$

\section{E. General Remarks}

Having multipath or shadow fading shifts the distribution of $I_{A}$ away from Gaussianity. To maintain Gaussianity, the density of active nodes should be increased, or the exclusion region should be extended. Fig. 6 reflects the divergence from the Gaussian distribution that the fading distribution may cause. It shows three different fading scenarios. It is clear from the figure that the divergence caused by lognormal shadow fading with $\sigma_{s}=6 \mathrm{~dB}$ is more than that caused by Rayleigh multipath fading. In the presence of significant shadow fading, the Gaussian distribution is a very poor approximation of the distribution of 
$I_{A}$ unless the active node density is too high, or the exclusion region is too wide.

\section{CONCLUSION}

The emergence of different types of wireless networks has promoted an interest in characterizing the distribution of the aggregate interference power in large wireless networks. In this paper, we have studied the convergence of this distribution to a Gaussian distribution. Based on the Berry-Esseen bound, we cast, in a single mathematical framework, some observations scattered across the literature about the Gaussianity of the distribution of the aggregate interference power. We showed that an increase in the size of the exclusion region brings the distribution of the aggregate interference power closer to the Gaussian distribution. Increasing the active node density has a similar effect. However, the convergence is faster with the increase in the size of the exclusion region compared with the increase in the active node density. In contrast, channel fading causes divergence from Gaussianity. Shadow fading typically causes more divergence, as compared with multipath fading.

\section{APPENDIX A \\ BERRY-ESSEEN THEOREM}

The following theorem describes the Berry-Esseen bound in its basic form.

Theorem 1 (Berry-Esseen Theorem [19]): Let $\left\{Y_{j}\right\}$ be independent random variables with a common $\operatorname{cdf} F$, with zero mean, nonzero variance $\left(\sigma^{2}>0\right)$, and finite third absolute moment $\left(\rho=\mathbb{E}\left[\left|Y_{j}\right|^{3}\right]<\infty\right)$. Then, for all $y$ and $k$

$$
\left|F_{k}(y)-F_{N}(y)\right| \leq \frac{3 \rho}{\sigma^{3} \sqrt{k}}
$$

where $F_{k}$ is the cdf of the normalized sum $Z=$ $(1 / \sigma \sqrt{k}) \sum_{j=1}^{k} Y_{j}$, and $F_{N}$ is the cdf of the standard normal distribution, i.e., $\mathcal{N}(0,1)$.

Proof: The proof can be found in [19, p. 543].

According to [19], the factor 3 on the right is not claimed to be the optimal value. Therefore, it is expected that different references may propose smaller values.

\section{APPENDIX B \\ Derivation of THE Cumulant-Based BERRY-ESSEEN BOUND}

The shot noise considered in [11] is assumed to be generated by a stationary marked point process defined in $\mathbb{R}^{d} \times \mathbb{K}$, where $\mathbb{R}^{d}$ is a $d$-dimensional Euclidean space, and $\mathbb{K}$ is a space of marks. This shot noise has the form

$$
\Xi(t)=\sum_{i} f\left(t-q_{i}, x_{i}\right)
$$

where $f$ is a real-valued function denoting the effect at $t \in \mathbb{R}^{d}$ caused by an event at a random position $q_{i} \in \mathbb{R}^{d}$ with an independent random mark $x_{i} \in \mathbb{K}$. The marks $x_{i}$ are assumed to be mutually independent. According to [11, Th. 7], the
Berry-Esseen bound for the normalized shot noise $Z$ when the underlying process is a stationary PPP can be written as

$$
\left|F_{Z}(y)-F_{N}(y)\right| \leq 2.21 \frac{H_{3}}{\sqrt{\lambda}\left(H_{2}\right)^{3 / 2}}
$$

where $\quad H_{3}=\int_{\mathbb{R}^{d}} \mathbb{E}_{X}\left[\left|f\left(u, x_{i}\right)\right|^{3}\right] d u, \quad$ and $\quad H_{2}=$ $\int_{\mathbb{R}^{d}} \mathbb{E}_{X}\left[f^{2}\left(u, x_{i}\right)\right] d u$. Within the context of this paper, the function $f$ represents an individual interference power, which is positive. Therefore, $\mathrm{H}_{3}$ can be written without the absolute value function as $H_{3}=\int_{\mathbb{R}^{d}} \mathbb{E}_{X}\left[f^{3}\left(u, x_{i}\right)\right] d u$.

To relate $H_{3}$ and $H_{2}$ to the cumulants of the shot noise in (22), the characteristic function of $\Xi$ should be determined first. Based on Campbell's theorem [20], the characteristic function of the sum in (22), which is over a stationary and independently marked PPP, can be written as

$\phi(\omega)=\exp \left(\lambda \int_{\mathbb{R}^{d}} \int_{\mathbb{K}}(\exp (j \omega f(u, x))-1) f_{X}(x) d x d u\right)$

where $f_{X}$ is the pdf of $X$. From (24), the $m$ th cumulant can be obtained by

$\kappa_{m}=\frac{1}{j^{m}}\left[\frac{d^{m} \ln (\phi(\omega))}{d \omega^{m}}\right]_{\omega=0}=\lambda \int_{\mathbb{R}^{d}} \mathbb{E}_{X}\left[f^{m}(u, x)\right] d u$.

Comparing (25) with the expressions of $\mathrm{H}_{3}$ and $\mathrm{H}_{2}$ yields $\kappa_{2}=\lambda H_{2}$ and $\kappa_{3}=\lambda H_{3}$. Thus, the Berry-Esseen bound can be written in terms of the second and third cumulants as

$$
\left|F_{Z}(y)-F_{N}(y)\right| \leq 2.21 \frac{\kappa_{3}}{\kappa_{2}^{3 / 2}} .
$$

\section{APPENDIX C \\ Generalization OF THE BERRY-ESSEEN BOUND FOR A $d$-DIMENSIONAL SPACE}

Results reported in [11] and [15] for the Berry-Esseen bound are applicable for a multidimensional space. Therefore, we can use (3) to investigate the Gaussianity of the aggregate interference power of a wireless network deployed in a $d$-dimensional space. However, we need to evaluate cumulants of a $d$-dimensional space. To achieve this, we utilize cumulant expressions provided in [17], and we extend them to our problem. As a result, we have the following expression for the $m$ th cumulant in a $d$-dimensional space:

$$
\kappa_{m}\left(I_{A}\right)=\frac{d b_{d} \lambda}{m n-d} r_{o}^{d-m n} \tilde{\mu}_{m}(X)
$$

where $b_{d}$ is the volume of the unit ball in $\mathbb{R}^{d}$, which is equal to $\left(\pi^{d / 2} / \Gamma(1+(d / 2))\right)$, and $\Gamma(\cdot)$ is the gamma function. To avoid divergence of the interference, we assume that $n>d$. Using (3) and (27), the Berry-Esseen bound becomes

$$
\left|F_{Z}(y)-F_{N}(y)\right| \leq 2.21 \frac{(2 n-d)^{\frac{3}{2}}}{d^{\frac{1}{2}}(3 n-d)} \frac{1}{\sqrt{\lambda v_{d}\left(0, r_{o}\right)}} \frac{\tilde{\mu}_{3}(X)}{\left[\tilde{\mu}_{2}(X)\right]_{(28)}^{\frac{3}{2}}}
$$

where $v_{d}\left(0, r_{o}\right)=b_{d} r_{o}^{d}$, which is the volume of the exclusion region in a $d$-dimensional space. It is worth noting that the 
rate of the Gaussian convergence with respect to the changes in the node density and exclusion region is $\sqrt{\lambda v_{d}\left(0, r_{o}\right)}$. This quantity, i.e., $\sqrt{\lambda v_{d}\left(0, r_{o}\right)}$, reflects the average number of interferers in a volume equal to the volume of the exclusion region.

These expressions are applicable for $r_{o} \geq r_{c}$. Expressions for the other case, i.e., $r_{o}<r_{c}$, can be obtained in a similar approach.

\section{ACKNOWLEDGMENT}

The authors would like to thank S. Szyszkowicz from Carleton University for his helpful comments.

\section{REFERENCES}

[1] M. Aljuaid and H. Yanikomeroglu, "Investigating the validity of a Gaussian approximation for the distribution of the aggregate interference power in large wireless networks," in Proc. 25th Biennial Symp. Commun., Kingston, ON, Canada, May 2010, pp. 122-125.

[2] R. K. Ganti and M. Haenggi, "Interference in ad hoc networks with general motion-invariant node distribution," in Proc. IEEE ISIT, Toronto, ON, Canada, Jul. 2008, pp. 1-5.

[3] M. Haenggi, J. G. Andrews, F. Baccelli, O. Dousse, and M. Franceschetti, "Stochastic geometry and random graphs for the analysis and design of wireless networks," IEEE J. Sel. Areas Commun., vol. 27, no. 7, pp. 1029-1046, Sep. 2009.

[4] A. Ghasemi and E. S. Sousa, "Interference aggregation in spectrumsensing cognitive wireless networks," IEEE J. Sel. Topics Signal Process., vol. 2, no. 1, pp. 41-56, Feb. 2008.

[5] M. Z. Win, P. C. Pinto, and L. A. Shepp, "A mathematical theory of network interference and its applications," Proc. IEEE, vol. 97, no. 2, pp. 205-230, Feb. 2009.

[6] C. C. Chan and S. V. Hanly, "Calculating the outage probability in a CDMA network with spatial Poisson traffic," IEEE Trans. Veh. Technol., vol. 50, no. 1, pp. 183-204, Jan. 2001.

[7] J. S. Evans and D. Everitt, "On the teletraffic capacity of CDMA cellular networks," IEEE Trans. Veh. Technol., vol. 48, no. 1, pp. 153-165, Jan. 1999.

[8] L. Le Cam, "The central limit theorem around 1935," Stat. Sci., vol. 1, no. 1, pp. 78-91, Feb. 1986.

[9] A. Hasan and J. G. Andrews, "The guard zone in wireless ad hoc networks," IEEE Trans. Wireless Commun., vol. 6, no. 3, pp. 897-906, Mar. 2007.

[10] R. Menon, R. Buehrer, and J. Reed, "On the impact of dynamic spectrum sharing techniques on legacy radio systems," IEEE Trans. Wireless Commun., vol. 7, no. 11, pp. 4198-4207, Nov. 2008.

[11] L. Heinrich and V. Schmidt, "Normal convergence of multidimensional shot noise and rates of this convergence," Adv. Appl. Prob., vol. 17, no. 4, pp. 709-730, Dec. 1985

[12] E. S. Sousa and J. A. Silvester, "Optimum transmission ranges in a directsequence spread-spectrum multihop packet radio network," IEEE J. Sel. Areas Commun., vol. 8, no. 5, pp. 762-771, Jun. 1990.

[13] H. Inaltekin, M. Chiang, H. V. Poor, and S. B. Wicker, "On unbounded path-loss models: Effects of singularity on wireless network performance," IEEE J. Sel. Areas Commun., vol. 27, no. 7, pp. 1078-1091, Sep. 2009.

[14] J. W. Gluck and E. Geraniotis, "Throughput and packet error probability in cellular frequency-hopped spread spectrum radio networks," IEEE $J$. Sel. Areas Commun., vol. 7, no. 1, pp. 148-160, Jan. 1989.

[15] J. A. Lane, "The Berry-Esseen bound for the Poisson shot-noise," Adv. Appl. Prob., vol. 19, no. 2, pp. 512-514, Jun. 1987.

[16] M. Aljuaid and H. Yanikomeroglu, "A cumulant-based characterization of the aggregate interference power in wireless networks," in Proc. IEEE VTC-Spring, Taipei, Taiwan, May 2010, pp. 1-5.
[17] S. Srinivasa and M. Haenggi, "Modeling interference in finite uniformly random networks," in Proc. Int. Workshop Inf. Theory Sens. Netw., Santa Fe, NM, Jun. 2007.

[18] P. M. Shankar, "Performance analysis of diversity combining algorithms in shadowed fading channels," Wireless Pers. Commun., vol. 37, no. 1/2, pp. 61-72, Apr. 2006

[19] W. Feller, An Introduction to Probability Theory and Its Applications, 2nd ed. New York: Wiley, 1971.

[20] J. F. C. Kingman, Poisson Processes. New York: Oxford Univ. Press, 1993.

Muhammad Aljuaid received the B.Sc. and M.Sc. degrees in electrical engineering from King Fahd University of Petroleum and Minerals, Dhahran, Saudi Arabia, in 1994 and 1999, respectively. He is currently working toward the Ph.D. degree with Carleton University, Ottawa, ON, Canada.

In November 1995, he joined Saudi Aramco, Dhahran, where he was actively involved in many technical projects related to wireless networks and control systems. He obtained some certifications in the area of wireless networking, such as Certified Wireless Network Administrator (CWNA) and Certified Wireless Security Professional (CWSP). Moreover, he is a holder of an amateur radio operator license. His research interests include wireless sensor networks, interference characterization, and spectrum sharing in large wireless networks.

Mr. Aljuaid was a recipient of a scholarship from Saudi Aramco in 2005.

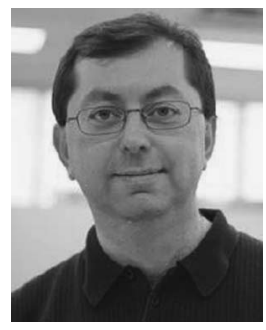

Halim Yanikomeroglu (M'08) received the B.Sc. degree in electrical and electronics engineering from Middle East Technical University, Ankara, Turkey, in 1990 and the M.A.Sc. degree in electrical engineering (now electrical and computer engineering) and the Ph.D. degree in electrical and computer engineering from the University of Toronto, Toronto, ON, Canada, in 1992 and 1998, respectively.

He was with the R\&D Group, Marconi Kominikasyon A.S., Ankara, from 1993 to 1994. Since 1998, he has been with the Department of Systems and Computer Engineering, Carleton University, Ottawa, ON, where he is currently a Full Professor with tenure. $\mathrm{He}$ is an Adjunct Professor with Prince Sultan Advanced Technologies Research Institute, King Saud University, Riyadh, Saudi Arabia. He has been involved in the steering committees and technical program committees of numerous international conferences; he has also given 18 tutorials at such conferences. His research interests cover many aspects of the physical, medium-access, and networking layers of wireless communications, with a special emphasis on multihop/relay/mesh networks and cooperative communications. His research is currently funded by Samsung Advanced Institute of Technology (Korea), Huawei (China), the Communications Research Centre of Canada, Research in Motion (Canada), and the Natural Sciences and Engineering Research Council of Canada.

Dr. Yanikomeroglu is a Registered Professional Engineer in the Province of Ontario. He is a member of the Steering Committee of the IEEE Wireless Communications and Networking Conference (WCNC) and has been involved in the organization of this conference over the years, including serving as the Technical Program Cochair of WCNC 2004 and the Technical Program Chair of WCNC 2008. He is the General Cochair of the IEEE Vehicular Technology Conference to be held in Ottawa in September 2010 (VTC 2010-Fall). He was an Officer of IEEE's Technical Committee on Personal Communications (Chair in 2005-2006, Vice Chair in 2003-2004, and Secretary in 2001-2002). He was also a member of the IEEE Communications Society's Technical Activities Council (2005-2006). He was an Editor for IEEE TRANSACTIONS ON WiReless COMMUNiCATIONS (2002-2005) and IEEE COMMUNICATIONS SURVEYS AND TUTORIALS (2002-2003) and a Guest Editor for the Wiley Journal on Wireless Communications and Mobile Computing. He was a recipient of the Carleton University Research Achievement Award 2009. 